\title{
MECHANISM OF ELECTRICAL CONDUCTIVITY IN METALLIC FIBER-BASED YARNS
}

\author{
Juan $\mathrm{Xie}^{1}$, Menghe $\mathrm{MiaO}^{2}$, Yongtang $\mathrm{Jia}^{1}$ \\ ${ }^{1}$ School of Textile Materials and Engineering, Wuyi University, Jiangmen, Guangdong Province, China \\ ${ }^{2}$ CSIRO Manufacturing, 75 Pigdons Road, Waurn Ponds, Victoria 3216, Australia \\ yongtjh@163.com
}

\begin{abstract}
:
We explore the conductive mechanism of yarns made from metallic fibers and/or traditional textile fibers. It has been proposed for the first time, to our knowledge, that probe span length plays a great role in the conductivity of metallic fiber-based yarns, which is determined by the probability and number of conductive fibers appearing on a cross section and their connecting on two neighboring sections in a yarn's longitudinal direction. The results demonstrate that yarn conductivity is negatively influenced to a large extent by its length when metallic fibers are blended with other nonconductive materials, which is beyond the scope of conductivity theory for metal conductors. In addition, wicking and wetting performances, which interfere with fiber distribution and conductive paths between fibers, have been shown to have a negative influence on the conductivity of metallic fiber-based yarns with various structures and composed of different fiber materials. Such dependence of the conductivity on the probe span length, as well as on the moisture from air and human body, should get attention during investigation of the conductivity of metallic fiber-based composites in use, especially in cases in which conductive yarns are fabricated into flexible circuit boards, antennas, textile electrodes, and sensors.
\end{abstract}

\section{Keywords:}

conductivity, metallic fibers, conductive yarn, wicking, wetting

\section{Introduction}

The replacement of electronic devices by electrically conductive fibrous materials plays an enormous role in the development of flexible, lightweight, launderable, and wearable e-textiles [1], which are applied in physiological sensing [2], flexible circuits [3], electromagnetic shielding [4], radiofrequency identification tags [5], and energy storage [6]. E-textiles can be made from conductive materials, such as metal micropowder [7], metallic fiber [8], graphene [9], carbon nanotubes (CNTs) [10, 11], and intrinsically conducting polymers, using hybrid spinning [12, 13] and coating techniques [14]. Some studies have found that the conductivity of composites based on CNTs, hybrid carbon/polymer [15], and particle fillers [16] is influenced by the measurement direction [17] and the attributes of conductive fillers such as the aspect ratio [18], size, shape [19], and conductivity [20]. As a major category of conductive composites, however, metallic fiber-based conductive yarns have been rarely considered in terms of their conductive mechanism. In our previous work [21], we have reported that the probe span length measuring the electrical resistance of metallic fiber-blended yarns is a key factor influencing conductive composites' percolation threshold and critical exponent.

In this study, the effect of the probe span length of yarn on its conductivity is further explored and the mechanism of this distinctive electric character is interpreted from the perspective of fiber distribution and the possibility of formation of conductive paths between conductive fibers. The results indicate that the conductivity of metallic fiber-based yarns is negatively influenced to a great extent by its length, which is beyond the scope of the electric conductivity principle for metallic conductors. The reason is then reported for the first time and effectively proven. In addition, due to the fact that moisture from the environment and human body is a key factor affecting the properties of polymer materials and smart clothing made from conductive yarns, the wicking and wetting performances of conductive yarns are measured to simulate and analyze their effect on the conductivity of metallic fiberbased yarns. The conductive mechanism is again proved by the result that the wicking and wetting performances, which affect fiber distribution and conductive path formation, bring about changes in the conductivity of yarns.

\section{Experimental}

Metallic fiber-based conductive blended yarns were made from cotton fibers of average length $3.2 \mathrm{~cm}$ and stainless steel (SS) fibers of diameter $8 \mathrm{~mm}$ and average length $5 \mathrm{~cm}$. Several distinctive SS fiber weight ratios were selected as follows: $10 \%$ (10\% SS fibers blended with $90 \%$ cotton fibers); $15 \%$ and $20 \%$ for conductive blended yarns; and $100 \%$ for pure conductive yarn. Their measured average linear densities were 40 tex $(\mathrm{mg} / \mathrm{m}), 42$ tex, 42 tex, and 50 tex, respectively. As a control group, a commercial silver-coated nylon multifilament yarn (14 tex) with electrical resistance $5 \mathrm{~W} / \mathrm{cm}$ was subjected to similar tests to explore the influence of various yarn structures on their conductive performance. 
The electrical resistances of the yarn samples were measured with a two-probe method using a digital multimeter (DIGITECH QM1571), shown in Figure 1(a). A constant load (32 g) was applied on each end of the yarn sample, with the probe span length $L$ (the distance between two measurement pens) equaling $5 \mathrm{~cm}, 10 \mathrm{~cm}, 25 \mathrm{~cm}$, and $50 \mathrm{~cm}$, respectively. Thirty specimens were prepared from each yarn sample. Then, the average specific conductivity $s_{s p}$ was obtained from the experimental resistance and the linear density of each specimen.

Two groups of conductive yarns were selected for the wicking and wetting tests, as shown in Figure $1(b)$. The first group contained silver-coated nylon yarn and $20 \%$ SS/cotton blended yarn with different yarn geometries, and the other group contained $20 \%$ SS/cotton blended yarn and $25 \%$ SS/ PP $(25 \%$ SS short fibers blended with $75 \%$ polypropylene [PP] short staple fibers) due to the varying hydrophilicity values of the component fibers. Wicking and wetting performances were measured by dripping water on the straight and relaxed yarn samples of length about $5 \mathrm{~cm}$. Meanwhile, the real-time resistance of yarns was recorded by a digital multimeter RIGOL DM3068 and displayed on a computer. The test time was set as follows: 1 hour for silver-coated conductive filaments and $25 \%$ SS/PP; and 2 hours for $20 \%$ SS/cotton due to the hydrophilicity of cotton fibers. All above-mentioned tests were carried out under standard atmospheric conditions with temperature of $20^{\circ} \mathrm{C}$ and humidity of $65 \%$.

\section{Conductive mechanism}

Yarns made from conductive fibers and/or nonconductive materials will conduct electricity only when conductive fibers exist on every cross section of yarns, and connection between the conductive fibers must occur in the lengthwise direction of the yarn. The conductivity of yarns of certain length is determined by the conductivity of the conductive fillers and the possibility of formation of conductive paths, of which the latter mainly depends on the number of conductive fibers on any cross section and the number of connections between conductive fibers on two adjacent sections. Thus, the conductivity of yarns of length $L$ is determined by the probabilities of conductive fibers appearing on any section and thereafter connecting on two neighboring sections, as well as by the corresponding number of fibers and number of connections.

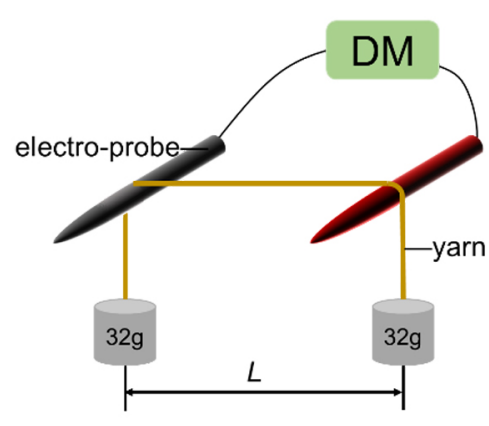

(a)

\subsection{Probability of conductive fiber appearing on any cross section of yarn}

It is assumed that fibers have ideal circular cross sections and are evenly distributed in the lengthwise direction of yarn. The number of SS fibers $n$ and the total number of fibers $N$ in a yarn of unit length $\Delta L$, where $\Delta L(\mathrm{~cm})$ is shorter than the length of both fibers (SS fiber length: $5 \mathrm{~cm}$; cotton fiber length: $3.2 \mathrm{~cm}$ ), with the length contraction of fibers due to twist being ignored, can be calculated using the following expression:

$$
\left\{\begin{array}{c}
n=\frac{N_{t} \cdot r}{N_{t s s}} \\
N=\frac{N_{t} \cdot r}{N_{t s s}}+\frac{N_{t} \cdot(1-r)}{N_{\text {tcotton }}}
\end{array}\right.
$$

where $N_{\mathrm{t}}$ (tex), $N_{\text {tss }}\left(0.41\right.$ tex), and $N_{\text {tcotton }}(0.17$ tex $)$ are the linear densities of blended yarn, SS fiber, and cotton fiber, respectively, and $r(\%)$ is the weight ratio (blend ratio) of SS fibers in the yarn.

The probability of conductive SS fiber appearing on a yarn's cross section is related to the ratio of conductive fillers. Its number on cross section $X$ follows the binomial distribution. The probability $p$ of success of conduction by the conductive fiber is determined by the ratio between the number $n$ of conductive fibers and the number $N$ of all fibers. The probability of getting exactly $k S S$ fibers in total number of $n$ is given by the probability mass function:

$$
P_{r}(X=k)=\frac{n !}{k !(n-k) !} p^{k}(1-p)^{n-k}
$$

Based on binomial distribution, $k$ is set to be the expected number (average number) of successful results, and we have

$$
k=E[X]=n p=n \cdot \frac{n}{N}
$$

\subsection{Connecting probability between and number of conductive fibers in two adjacent sections}

Figure 2 shows the longitudinal trajectories of SS fibers in a blended yarn of length $L$ (cotton fibers are not displayed here), where, in the cross section of the yarn, the SS fibers may (i) appear and connect with other fiber(s), (ii) appear but not connect, or (iii) not appear at all.

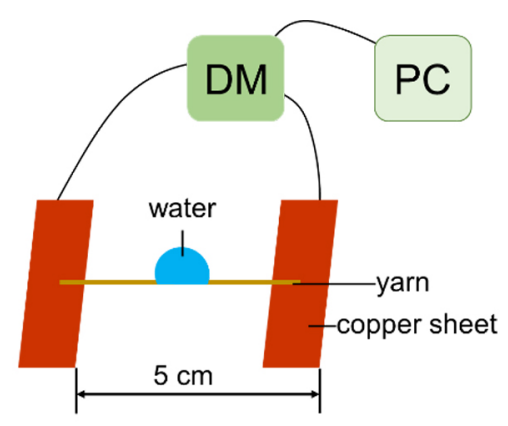

(b)

Figure 1. Measurement diagrams: (a) electrical resistance test of conductive yarns with probe span length $L$ and (b) wicking and wetting tests. 
Connection between conductive fibers on two neighboring sections enables the flow of electric current through the fibers and makes the yarn conductive. Therefore, it is assumed that there are $i$ pairs of neighboring sections along the yarn of length $L$, where $i=L / \Delta L$. The expected number of conductive fibers appearing on any section is $k$, and $k_{\mathrm{ci}}\left(n^{3} k^{3} k_{\mathrm{c} 1}{ }^{3} k_{\mathrm{c} 2}{ }^{31} /{ }^{3} k_{\mathrm{ci}}\right.$ ${ }_{1}^{3} k_{\mathrm{ci}}{ }^{3} 0$ ) is the expected number of connecting conductive fibers in the $i^{\text {th }}$ adjacent sections, which also follows the binomial distribution. According to Equations (2) and (3), the probability of getting exactly $k_{\mathrm{ci}}$ successes in $k_{\mathrm{c} \text { - } 1}$ trials can be deduced as in Equation 4:

$\left\{\begin{array}{ccc}P_{r 1}\left(\xi=k_{c 1}\right)=C_{k}^{k_{c 1}}\left(\frac{k}{N}\right)^{k_{c 1}}\left(1-\frac{k}{N}\right)^{\left(k-k_{c 1}\right)}, & k_{c 1}=k \cdot \frac{k}{N}=\left(\frac{n}{N}\right)^{3} \cdot n \\ P_{r 2}\left(\xi=k_{c 2}\right)=C_{k_{c 1}}^{k_{c 2}}\left(\frac{k}{N}\right)^{k_{c 2}\left(1-\frac{k}{N}\right)^{\left(k_{c 1}-k_{c 2}\right)},} & k_{c 2}=k_{c 1} \cdot \frac{k}{N}=\left(\frac{n}{N}\right)^{5} \cdot n \\ \vdots & \vdots \\ P_{r i}\left(\xi=k_{c i}\right)=C_{k_{c i-1}}^{k_{c i}}\left(\frac{k}{N}\right)^{k_{c i}}\left(1-\frac{k}{N}\right)^{\left(k_{c i-1}-k_{c i}\right)}, & k_{c i}=k_{c i-1} \cdot \frac{k}{N}=\left(\frac{n}{N}\right)^{(2 i+1)} \cdot n\end{array}\right.$

Then, the resistance of yarn $R_{\mathrm{y}}$ of length $L$, shown in Figure 2(c), is determined by the number of parallel resistances of SS fibers in the radial direction of yarn of unit length $\Delta L$ and the number of series resistance of the yarn segments, as shown in Equation (5).

$R_{y} \propto \frac{R_{s s}}{k} \cdot \frac{\Delta L}{2}+\frac{R_{s s} \cdot \Delta L}{k_{c 1}}+\frac{R_{s s} \cdot \Delta L}{k_{c 2}}+\ldots+\frac{R_{s s} \cdot \Delta L}{k_{c i-1}}+\frac{R_{s s} \cdot \Delta L}{k_{c i}}+\frac{R_{s s}}{k_{c i}} \cdot \frac{\Delta L}{2}$

where the constant $R_{\mathrm{ss}}(\mathrm{W} / \mathrm{cm})$ is the resistance per unit length of SS fiber and $R_{y}(\mathrm{~W})$ is the resistance of the conductive yarn of length $L(\mathrm{~cm})$.

\section{Results and discussion}

\subsection{Pure metallic fiber-based conductive yarns}

Table 1 shows the values of the component fibers of various yarns. For a conductive yarn made from $100 \%$ SS short fibers $(r=100 \%)$, the values of fiber numbers $n$ and $N$ are equal, which enables $n=k=k_{\mathrm{c} 1}=k_{\mathrm{c} 2}=1 / 4=k_{\mathrm{ci}-1}=k_{\mathrm{ci}}$. According to Equation (5), we have

$$
\left\{\begin{array}{l}
R_{y} \propto \frac{R_{s s}}{n} \cdot L \\
\sigma_{s p} \propto \frac{n}{R_{s s} N_{t}}
\end{array}\right.
$$

Therefore, the specific conductivity $\mathrm{s}_{\mathrm{sp}}\left(\mathrm{S} \mathrm{cm}^{2} / \mathrm{g}\right)$ [21] of pure SS fiber-based conductive yarn with certain linear density $N_{t}$ is considered to be irrelevant to yarn length $L$.

Table 1. Fiber numbers of all conductive yarns

\begin{tabular}{|c|c|c|c|c|}
\hline \multirow{2}{*}{$\mathbf{r}(\%)$} & \multicolumn{2}{|c|}{ Theoretical values } & \multicolumn{2}{c|}{ Measured values } \\
\cline { 2 - 5 } & $n$ & $N$ & $n$ & $N$ \\
\hline 10 & 10 & 221 & 11 & 239 \\
\hline 15 & 15 & 226 & 14 & 262 \\
\hline 20 & 20 & 218 & 21 & 256 \\
\hline 100 & 122 & 122 & 114 & 114 \\
\hline
\end{tabular}

$L$

(a)

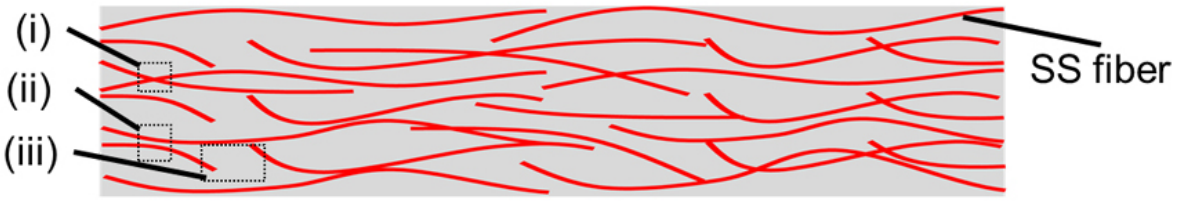

(b)

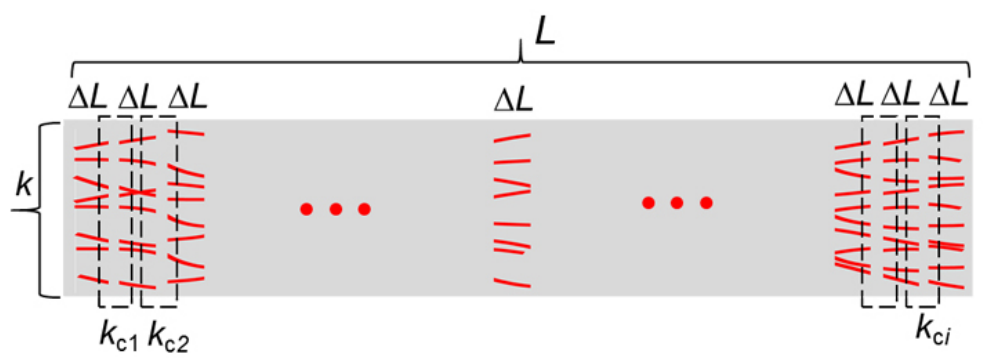

(c)

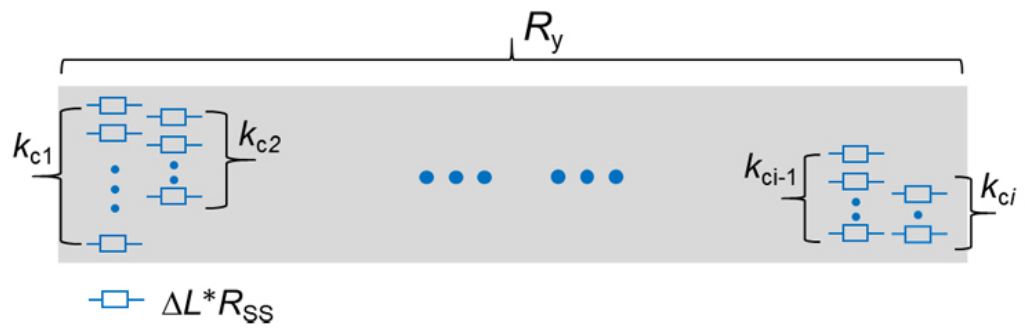

Figure 2. (a) Longitudinal trajectories of SS fibers in blended yarn of length $\Delta L$, (b) number of SS fibers connecting between two adjacent sections, and (c) yarn resistance including series and parallel unit resistances. 
Figure 3 shows the average specific conductivity and standard deviation of pure SS fiber-based conductive yarn with different yarn lengths $L$. It can be found that the overall trend of yarn conductivity is an approximately horizontal line of very large length, which indicates little effect of probe span length on the yarn's conductivity. However, it should be noted that conductivity at small length is slightly smaller than the average value, which is possibly attributed to the high unevenness of yarn at small length and to electrical testing error with low yarn resistance at short length.

As a control group, the conductivity of silver-coated nylon filament assembles was also investigated to prove that probe span length played a negligible role in the conductivity of metallic fiber-based yarn, where the number of conductive fiber $n$ equals the total fiber number $N$, and that the numbers of contacting conductive fibers $k_{\mathrm{ci}}$ on all cross sections are similar. In Figure $3 b$, the specific conductivity of metal-coated fibrous materials remains stable at various probe span lengths, indicating that there is no effect of probe span length on the conductive particle-coated yarn.

\subsection{Metallic fiber-based conductive blended yarns}

When the blend ratio $r$ is less than $100 \%$, the ratio between fiber numbers $n$ and $N$ is smaller than 1 . Then, the number of SS fibers connecting between two neighboring sections in Equation (4) would show a downward trend with an increment of $i$, i.e., $n>k>k_{\mathrm{c} 1}>k_{\mathrm{c} 2}>1 / 4>k_{\mathrm{ci}-1}>k_{\mathrm{ci}}>0$. As the probe span length increases, there are more pairs of adjacent sections, and the parallel resistance element $\frac{R_{s s} \cdot \Delta L}{k_{c i}}$ in Equation (5) gradually grows to an infinitely large value, resulting in descending specific conductivity and a nonlinear relationship between $R_{\mathrm{y}}$ and $L$.

Figure 4 shows the specific conductivities of metallic fiberbased blended yarns. More SS fibers in blended yarn lead to a higher possibility for their appearance and connection on any cross section, resulting in more conductive paths in the yarns and better conductive performance. But all the specific conductivity values are quite lower than that in pure SS fiber-based yarn in Figure 3(a). In addition, all conductivities exhibit downward trends as the probe span length increases.
The smaller the ratio of conductive fibers is, the larger is the decrement of conductivity.

From the above results of conductivity analysis of conductive yarns, the probe span length has been proven to have a great effect on the electrical conductivity of metallic fiber-based conductive blended yarns while having little influence on pure metallic fibrous materials. It is also confirmed that the conductive mechanism of metallic fiber-based materials is based on the probability and number of conductive fillers appearing on any cross section and connecting between two adjacent sections.

\subsection{Wicking and wetting performances of metallic fiber- based conductive yarns}

Figure 5 shows the wicking and wetting performances of metallic fiber-based conductive yarns. It can be found that the rate of water being drawn into yarn is the highest for SS/ PP yarn, while the other two yarns show little water-drawing ability. Obviously, the advantage in terms of surface energy in the case of cotton fibers relative to PP fibers (200 to $29 \mathrm{~mJ} / \mathrm{m}^{2}$ ) [22], which determines the wicking rate of fibrous materials, plays no role in the yarn's wicking and wetting performances. On the other hand, the circular cross section of the PP fiber

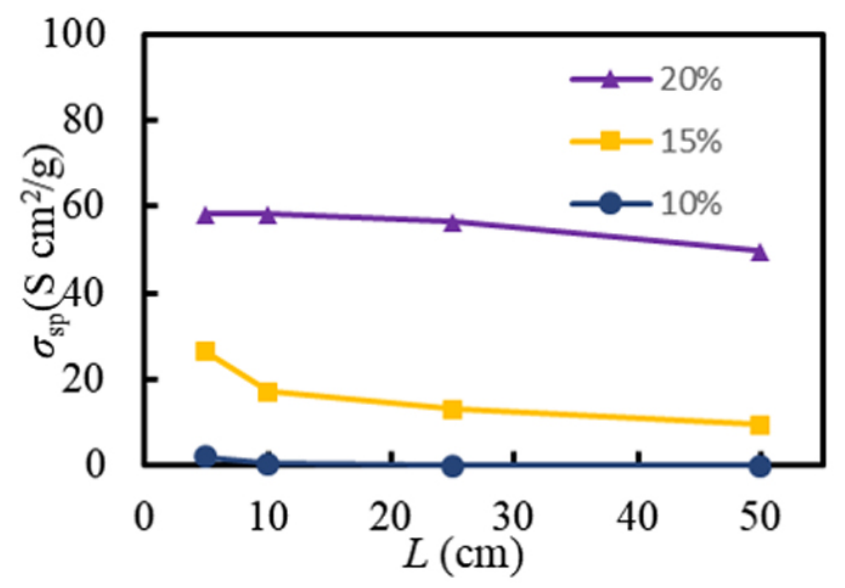

Figure 4. Specific conductivity of SS fiber-based conductive blended yarns with different probe span lengths.
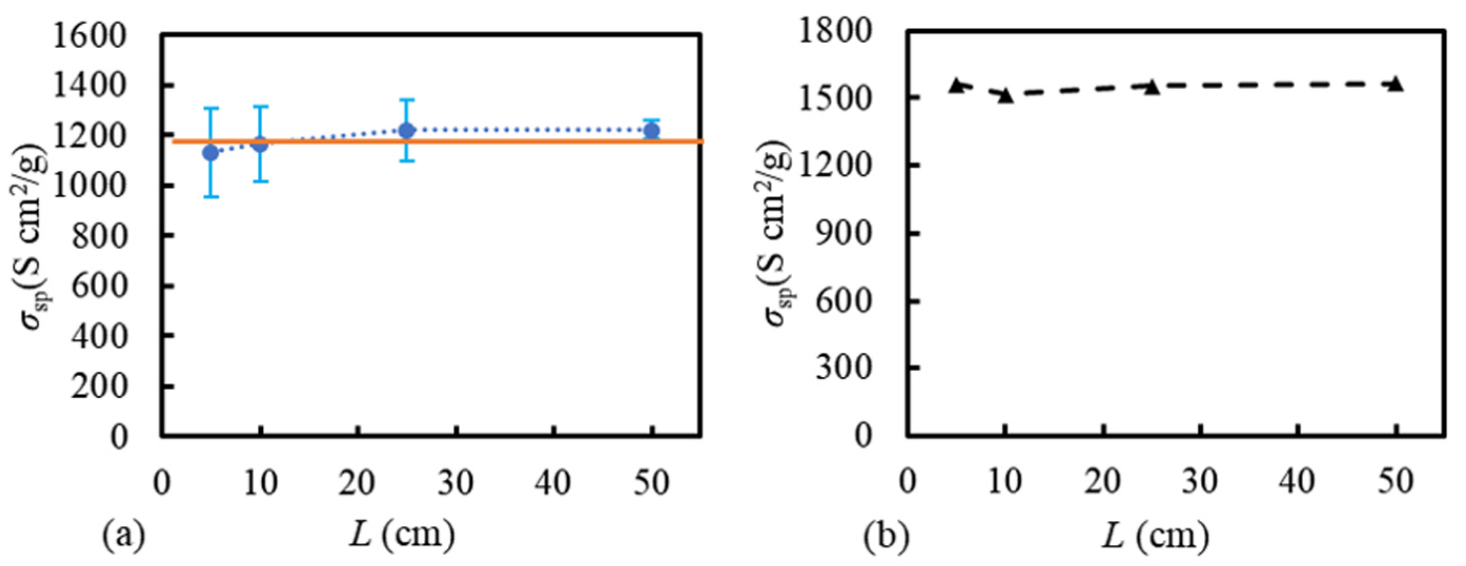

Figure 3. (a) Specific conductivity and standard deviation of $100 \%$ SS fiber-based conductive yarn; and (b) conductivity of silver-coated conductive filaments. 
lessens the formation of gaps between the fibers in an SS/PP yarn compared with the kidney-shaped cross section of cotton fibers, shown in Figure 5, probably leading to higher wicking ability and water-drawing rate [23]. As to the silver-coated conductive yarn, little gaps between filaments due to their tight arrangement and hydrophobic performance of the silver coating layer results in poor wicking and wetting abilities.

Figure 6 displays that yarns' conductivity shows various uptrends with wetting process. There is a slight increase of conductivity at the time of dripping of water on silver-coated yarn (Figure 6a), which may be caused by some purified water with ultra-low conductivity being drawn into the gaps between fibers. But the poor water-drawing ability and hydrophobicity of silver-coated filaments enable a horizontal line of conductivity with increments of the wetting time. On the other side, the conductivity of SS/PP and SS/cotton conductive blended yarns shows fluctuant increases and decreases. Probably, with the high rate of drawing of water into the SS/PP yarn, its cross section was enlarged and the connections between the SS fibers were affected. The resultant disconnecting and reconnecting of the conductive paths brought about more obvious ups and downs in conductivity for the SS/cotton blended yarn.
Theoretically, water molecules would be gradually absorbed by hydrophilic groups in cotton fibers, decreasing their mass specific resistance $(\mathrm{W} \ddot{\mathrm{Y}} \mathrm{g} / \mathrm{cm})$ from the $10^{-10}$ level at a relative humidity $(\mathrm{RH})$ of $10 \%$ to the $10^{-6}$ level at an $\mathrm{RH}$ of $80 \%$. Cotton fibers would transform from a nearly insulative situation into a slightly conductive state during the long moisture-absorbing process, which was set to 2 hours here. Therefore, the initial conductive network formed by the SS fibers would be inserted into some higher electric resistance (cotton fibers) in case of cohesiveness between component fibers, and the resistance of SS/PP blended yarn would see a larger overall ascending trend.

\section{Conclusion}

We have reported the conductive mechanism of metallic fiber-based conductive yarns. The influence of probe span length on yarn's conductivity was explored for the first time, the reasons of which were summarized as the probability and number of conductive fibers appearing on any cross section and connecting between two neighboring sections in the longitudinal direction of yarn. When yarn was made from

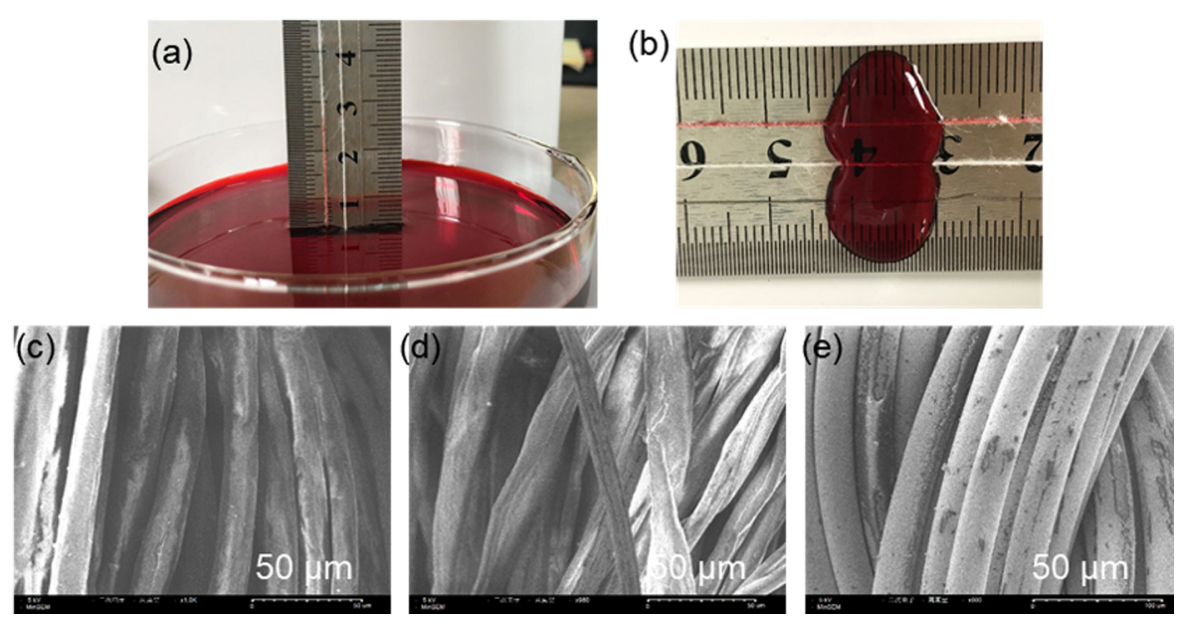

Figure 5. (a) Wicking performance; (b) wetting performance; and scanning electron microscopy images of conductive yarns, from left/top to right/ bottom are (c) $25 \%$ SS/PP, (d) $20 \%$ SS/cotton, and (e) silver-coated conductive yarns, respectively.
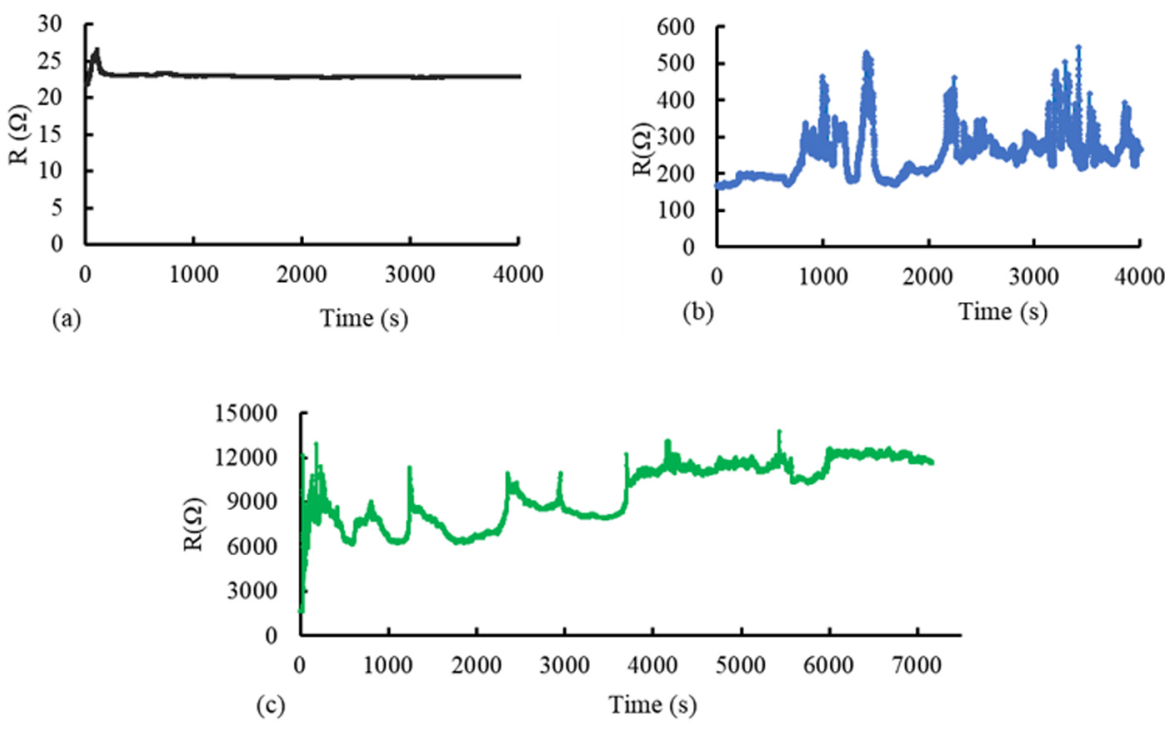

Figure 6. The resistance to water being absorbed by (a) silver-coated yarn, (b) $25 \%$ SS/PP, and (c) $20 \%$ SS/cotton conductive blended yarns. 
metallic short staple fibers as well as metal-coated filaments, its conductivity showed little change with increment of length, which is similar to that of metallic conductors, while yarn conductivity was negatively influenced to a large extent by probe span length when metallic fibers were blended with other nonconductive materials. The conductivity theory for metal conductors is not suitable in this case. Moreover, it was verified that the wicking and wetting performances, which interferes with fiber distribution and conductive paths between fibers, played a negative role in the conductivity of metallic fiberbased yarns with various structures and composed of different fiber materials. To sum up, influencing factors, such as probe span length and moisture from air and human body, should get attention in studies on the conductivity of metallic fiber-based composites in use, especially in cases wherein conductive yarns are fabricated into flexible circuit boards, antennas, textile electrodes, and sensors.

\section{Acknowledgments}

We acknowledge the financial support from Guangdong Province Ordinary University Youth Innovative Talents Project (Natural Science) (2016KQNCX173) and Youth Foundation of Wuyi University (2016zk01).

\section{References}

[1] Zeng, W., Shu, L., Li, Q., Chen, S., Wang, F., Tao, X. M. (2014). Fiber-based wearable electronics: a review of materials, fabrication, devices, and applications. Advanced Materials, 26, 5310-5336.

[2] Yamada, T., Hayamizu, Y., Yamamoto, Y., Yomogida, Y., Izadi-Najafabadi, A., Futaba, D. N., et al. (2011). A stretchable carbon nanotube strain sensor for humanmotion detection. Nature Nanotechnology, 6, 296-301.

[3] Park, M., Im, J., Shin, M., Min, Y., Park, J., et al. (2012). Highly stretchable electric circuits from a composite material of silver nanoparticles and elastomeric fibres. Nature Nanotechnology, 7, 7.

[4] Cheng, K. B., Ramakrishna, S., Lee, K. C. (2000). Electromagnetic shielding effectiveness of copper glass fiber knitted fabric reinforced polypropylene composites. Composites Part A: Applied Science and Manufacturing, 31, 1039-1045.

[5] Koski, K., Vena, A., Sydänheimo, L. (2013). Design and implementation of electro-textile ground planes for wearable UHF RFID patch tag antennas. IEEE Antennas and Wireless Propagation Letters, 12, 4.

[6] Liu, N., Ma, W., Tao, J., Zhang, X., Su, J., Li, L., et al. (2013). Cable-type supercapacitors of three-dimensional cotton thread based multi-grade nanostructures for wearable energy storage. Advanced Materials, 25, 49254931.

[7] Maaroufi, A. (2004). Electrical resistivity of polymeric matrix loaded with nickel and cobalt powders. Journal of Materials Science, 39, 265-270.
[8] Šafářová, V., Militký, J. (2012). A study of electrical conductivity of hybrid yarns containing metal fibers. Journal of Materials Science and Engineering B, 2, 197-202.

[9] Li, X., Hua, T., Xu, B. (2017). Electromechanical properties of a yarn strain sensor with graphene-sheath/polyurethanecore. Carbon, 118, 686-698.

[10] Mutiso, R. M., Winey, K. I. (2015). Electrical properties of polymer nanocomposites containing rod-like nanofillers. Progress in Polymer Science, 40, 63-84.

[11] Zhang, D., Zhang, Y., Miao, M. (2014). Metallic conductivity transition of carbon nanotube yarns coated with silver particles. Nanotechnology, 25, 275702.

[12] Schwarz, A., Cuny, L., Hertleer, C., Ghekiere, F., Kazani, l., et al. (2011). Electrical circuit model of elastic and conductive yarns produced by hollow spindle spinning. Materials Technology, 26, 121-127.

[13] Xie, J., Long, H., Miao, M. (2016). High sensitivity knitted fabric strain sensors. Smart Materials and Structures, 25, 105008.

[14] Allison, L., Hoxie, S., Andrew, T. L. (2017). Towards seamlessly-integrated textile electronics: methods to coat fabrics and fibers with conducting polymers for electronic applications. Chem Commun (Camb), 53, 7182-7193.

[15] Motaghi, A., Hrymak, A., Motlagh, G. H. (2015). Electrical conductivity and percolation threshold of hybrid carbon/ polymer composites. Journal of Applied Polymer Science, 132.

[16] Cai, W.-Z., Tu, S.-T., Gong, J.-M. (2006). A physically based percolation model of the effective electrical conductivity of particle filled composites. Journal of Composite Materials, 40, 2131-2142.

[17] Behnam, A., Guo, J., Ural, A. (2007). Effects of nanotube alignment and measurement direction on percolation resistivity in single-walled carbon nanotube films. Journal of Applied Physics, 102, 044313.

[18] White, S. I., Mutiso, R. M., Vora, P. M., Jahnke, D., Hsu, S., et al. (2010). Electrical percolation behavior in silver nanowire-polystyrene composites: simulation and experiment. Advanced Functional Materials, 20, 27092716.

[19] Kyrylyuk, A. V., Hermant, M. C., Schilling, T., Klumperman, B., Koning, C. E., et al. (2011). Controlling electrical percolation in multicomponent carbon nanotube dispersions. Nature Nanotechnology, 6, 364-369.

[20] Otten, R. H., van der Schoot, P. (2011). Connectivity percolation of polydisperse anisotropic nanofillers. The Journal of Chemical Physics, 134, 094902.

[21] Xie, J., Gordon, S., Long, H., Miao, M. (2015). Electrical percolation of fibre mixtures. Applied Physics A, 121, 589595.

[22] Holcombe, B. (2008). Wool performance apparel for sport. Woodhead Publishing Limited, Cambridge.

[23] Francis W. Minor, Anthony M. Schwartz, E.A. Wulkow \& Buckles, L. C. (1959) The Migration of Liquids in Textile Assemblies, Part II: The Wicking of Liquids in Yams, Textile Research Journal. 29, 10. 